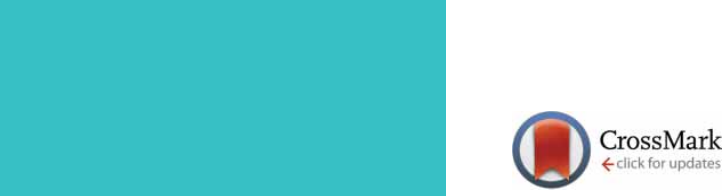

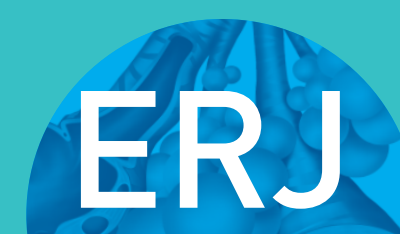

open research
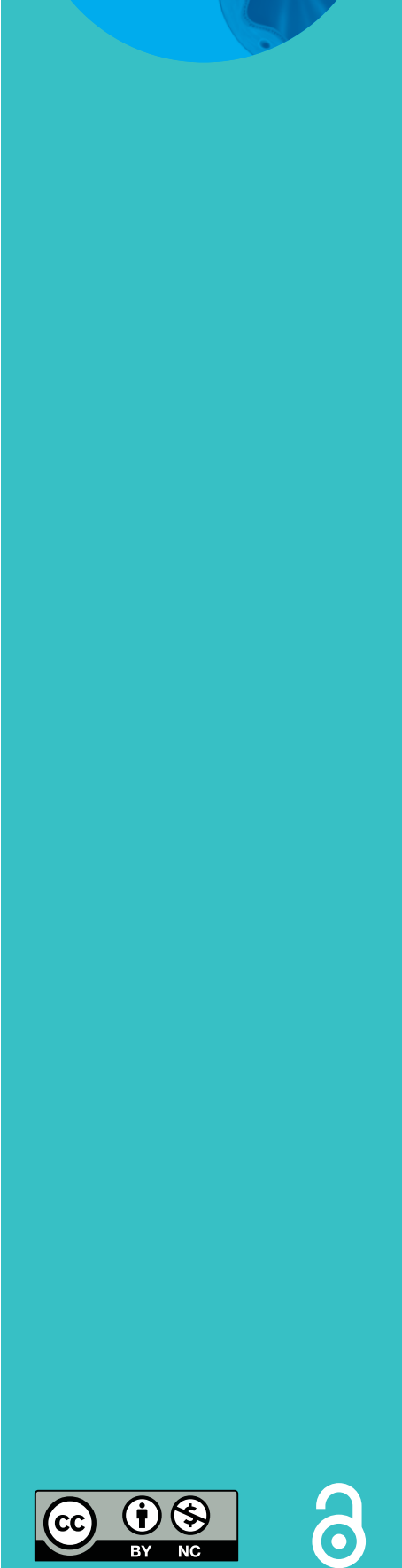

\section{ERS International Congress, Madrid, 2019: highlights from the Respiratory Infections Assembly}

\author{
Cristina Calarasu (1) ${ }^{1}$, Kiarina D. Chichirelo-Konstantynovych (10) ${ }^{2}$ and \\ Stefan Frent ${ }^{3}$
}

Affiliations: ${ }^{1}$ University of Medicine and Pharmacy of Craiova, Dept of Medical Specialities, Craiova, Romania. ${ }^{2}$ Dept of Infectious Diseases and Epidemiology, National Pirogov Memorial Medical University, Vinnytsya, Ukraine. ${ }^{3}$ Pulmonology Dept, University of Medicine and Pharmacy Timisoara, Timisoara, Romania.

Correspondence: Cristina Calarasu, University of Medicine and Pharmacy of Craiova, Dept of Medical Specialities, Petru Rares Street no. 2, Craiova Dolj 200349, Romania. E-mail: calarasu.cristinadyahoo.com.

ABSTRACT The European Respiratory Society (ERS) International Congress organised in Madrid, Spain, in 2019 welcomed $>22000$ participants from 134 countries. For each ERS assembly, an impressive number of abstracts were submitted. The topics covered by Assembly 10 (Respiratory Infections and Tuberculosis) were included this year in the top five research areas with the most submitted abstracts, with a total of 424 abstracts accepted for presentation. As it would be difficult for any delegate to stay up to date with all the scientific advances in the field, we wanted to highlight three of the Congress sessions that included presentations on respiratory infections and tuberculosis that we deemed as important and we hope the readers will consider this material of great interest.

@ERSpublications

The areas covered by @EuroRespSoc Assembly 10 were very well served at \#ERSCongress 2019: 219 abstracts that focused on respiratory infections and 205 on tuberculosis https://bit.ly/ 2XShcNs

Cite this article as: Calarasu C, Chichirelo-Konstantynovych KD, Frent S. ERS International Congress, Madrid, 2019: highlights from the Respiratory Infections Assembly. ERJ Open Res 2020; 6: 00316-2019 [https://doi.org/10.1183/23120541.00316-2019].

Received: 17 Nov 2019 | Accepted after revision: 15 April 2020

Copyright $\odot$ ERS 2020. This article is open access and distributed under the terms of the Creative Commons Attribution Non-Commercial Licence 4.0. 


\section{Fighting antimicrobial resistance: from prevention to antimicrobial stewardship}

The presentations included in this session focussed on the widespread clinical problem of antimicrobial resistance in respiratory diseases (community-acquired pneumonia (CAP) in particular) and on modern diagnostic approaches to overcome this sensitive issue.

Francesco Blasi discussed antimicrobial resistance in respiratory diseases and presented epidemiological data from the European Centre for Disease Prevention and Control [1]. Bacterial resistance was explained as a natural process caused by mutational alteration or incorporation of mobile genetic elements carrying resistance genes.

Clinical outcomes of CAP patients rely on the interaction between three main determinants: host, bacterium and drug [2]. Prof. Blasi argued that risk scores able to identify multidrug resistant (MDR) pneumonia upon hospital admission could inform patient treatment and prevent antibiotic misuse and complications. AlibERTI et al. [3] developed a score to evaluate the presence of MDR pathogens in patients with pneumonia coming from the community in two independent European cohorts of consecutive patients hospitalised with pneumonia in Barcelona, Spain, and Edinburgh, UK. The Aliberti score was better than the healthcare-associated pneumonia classification in predicting MDR pneumonia, especially when used in the intensive care unit setting [4]. Pneumonia caused by an MDR pathogen acquired in the community depends on patients' previous health status/comorbidities and previous contact with the healthcare system. Hospitalisation in the previous 90 days and residency in a nursing home or extended-care facility were found to be independent risk factors for pneumonia with a resistant pathogen and increased in-hospital mortality. These results support an individual evaluation of each patient in order to develop a targeted approach when selecting empiric antibiotic therapy [3].

Immunodepression state as a risk factor for MDR pneumonia was present in $18 \%$ of the population from an international, multicentre study enrolling adult patients with CAP requiring hospitalisation. The most common single risk factors for immunodeficiency were chronic steroid use (45\%), haematological malignancy $(25 \%)$ and chemotherapy (22\%). The authors of this study performed a multivariable regression analysis and found a strong association between Pseudomonas aeruginosa aetiology of pneumonia and a history of prior infection with Pseudomonas (OR 19.20, 95\% CI 11.71-31.50) or tracheostomy (OR 6.95, 95\% CI 2.87-16.85), and between Mycobacterium tuberculosis infection and malnutrition (OR 5.14, 95\% CI 2.21-11.93) [5]. Broad-spectrum antibiotic use was associated with increased mortality risk (OR 3.8, 95\% CI 2.5-5.9), a significant increase in length of hospital stay, greater cost and Clostridium difficile infection in another study recently published in the European Respiratory Journal [6].

Prof. Blasi concluded that improved infection control, widespread implementation of vaccination and reduction of antimicrobial consumption are efficient strategies to avoid development of antibiotic resistance.

Murat Akova, in his review "How to link antimicrobial and diagnostic stewardship", highlighted N. Fishman's data from the Hospital of the University of Pennsylvania (Philadelphia, PA, USA) suggesting that antimicrobial stewardship (AMS) is a key component of a multifaceted approach to prevent emergence of antimicrobial resistance [7]. This is a result of patients' and healthcare workers' proper education, rapid diagnosis and infection control, use of vaccines, and development of strategies to minimise antimicrobial resistance.

Various authors recommend using antibiotics 1) in a timely fashion (delay in antimicrobial therapy increases mortality), 2) appropriately (inappropriate therapy leads to higher mortality) and 3) per pharmacokinetic/pharmacodynamic parameters, and 4) to de-escalate at the earliest opportunity and 5) to discontinue as soon as possible [8-16]. Several studies showed a strong positive correlation between antibiotic use and resistance [17]. Using microbiological rapid diagnostic tests, such as multiplex immunoassays, would optimise treatment and reduce unnecessary prescription of antibiotics by as much as $30-50 \%$ in Europe and the USA [18-20].

The report by KLEIN et al. [21] on antibiotic use in 76 countries over 16 years showed that the antibiotic consumption rate in low- and middle-income countries has been growing, and in some countries exceeded levels typically observed in high-income countries. Dr Akova underlined several necessary actions in the global fight against antimicrobial resistance: improved sanitation, increased access to clean water, good governance with increased public healthcare funds and a regulated private health sector [22].

Obtaining necessary and reliable rapid diagnostic tests is problematic due the impossibility of bacterial specific detection in $62 \%$ cases [23], bacterial-viral mixed infection in $7-10 \%$ cases [24] and prescription of antibiotics in viral CAP (41\% cases) [25] prior to specific diagnostics [26]. Several studies have demonstrated the important role of procalcitonin [27-30] and C-reactive protein [27] as biomarkers to 
guide antibiotic therapy. WitTermans [31] reports a positive association between the extent of microbiological testing and antibiotic alteration, defined as a narrowing of the antibiotic spectrum, switching to a different class of antibiotics or from dual to monotherapy and discontinuation of antibiotic treatment. Thus, improved targeted diagnostic tests in terms of accessibility and convenient use may lead to antibiotic avoidance [32].

Herman Goossens, in his presentation "The new era of diagnostics: will it ever start?", emphasised that diagnostic tests affect around $60-70 \%$ of all clinical decisions [33] and account only for $0.8 \%$ of total healthcare expenditure in Europe [34]. Nowadays, medical activities require modern mobile multifunctional outpatient devices, which are easy to use and may provide an accurate, reliable diagnosis. Rapid diagnostic tests are more frequently used in clinical trials; however, their use faces many problems: unclear function and performance characteristics, a schism between pharmaceutical and diagnostic companies, unclear exploitation, regulatory blind spots, and demand of pharmaceutical companies to perform tests outside of microbiology labs. Performance characteristics of rapid diagnostic tests to guide narrow-spectrum antibiotics depend on the disease and targeted organism, on the prevalence of the disease, and on how the test and results will be used. New rapid tests are still being confronted with detection limits that are higher than standard amplification-based tests. The current diagnostic business model, focussed on technology used, laboratory activity measures and complexity indicators, is antiquated. Psychological, social, economic, ethical and organisational barriers prevent the uptake and development of diagnostics for AMS [35-38].

Prof. Goossens concluded that in order to reach the desired "golden age" of diagnostic testing, a consensus is necessary regarding a set of needs and the technologies required to satisfy those needs.

In the final presentation of this session, Keertan Dheda gave the definition of AMS as minimising the use of antibiotics through organised programmes, thus improving safety and quality of patient care by reducing the emergence and spread of MDR [39]. The evidence of AMS effectiveness was demonstrated in several studies on reduction in use of antibiotics. Pooled percentage change of total antimicrobial consumption after the implementation of AMS programmes was $-19.1 \%$ (95\% CI $-30.1--7.5 \%$ ), there was a decrease of disease burden in terms of colonisation and infection with MDR pathogens, and reduced mortality [40-43].

An expert consensus approach was used to develop a set of core elements for global hospital AMS: 1) hospital management leadership (leadership commitment), 2) accountability and responsibilities (single leader and defined roles), 3) expertise in infection management, 4) education and practical training, 5) actions driving responsible antimicrobial usage, 6) monitoring and surveillance, and 7) reporting and feedback. The experts recommend a combination of front- and back-end strategies using an AMS team. Front-end strategy makes antibiotics available through an approval process and back-end strategy reviews antibiotics after they are prescribed.

In conclusion, bacterial resistance is an international emergency and implementing AMS is a necessity; the scientific evidence is there and the time for action is now [44].

\section{Recent developments and future perspectives in the diagnosis and treatment of tuberculosis}

The aim of this session was to identify new therapeutic approaches for two categories of tuberculosis (TB) patients at increased risk: those with HIV co-infection and those with MDR/extensively drug-resistant (XDR)-TB.

François-Xavier Blanc reviewed some of the models of integrative care for patients with TB and HIV/ AIDS. He discussed the three models of TB and HIV/AIDS services: standalone services with TB and HIV linked by referral, partially integrated services, and fully integrated services.

The most important identified areas requiring TB-HIV integration were 1) testing and counselling for HIV in all patients with TB, 2) intensified case finding for TB in HIV infected patients, 3) isoniazid preventive therapy for HIV-positive patients that screen TB negative, 4) the moment of antiretroviral therapy (ART) initiation for all TB-HIV co-infected patients, 5) cotrimoxazole therapy for TB-HIV co-infected patients, 6) enhanced retention strategies including the post-test counselling and use of community-based outreach workers, and 7) enhanced ART and TB treatment adherence strategies including the use of community care workers for adherence support and community-based management of selected patients [45].

The objectives of TB/HIV integrative departments are 1) to decrease TB and HIV transmission, 2) to decrease morbidity and mortality from HIV and TB or from other HIV-related illnesses, 3) to improve the efficiency of healthcare services, and 4) to create a patient-centred approach for management of co-infected patients. Since 2011, integrative care models have focussed on screening for TB symptoms, 
with an emphasis on cough. Screening for cough is carried out whenever a patient enters a medical unit, in the reception area [46]. HIV diagnosis, staging and other services are also offered in every clinic service delivery area.

ART supply is offered in other dedicated TB/ART service areas with the exception of prevention treatment for mother to child transmission included in ante-natal services. TB diagnosis and treatment are given in the TB/HIV service area.

Dr Blanc underlined the necessity of a fully integrated data management system, adopting the approach of "one patient, one appointment, one file and one data management system".

People living with HIV in resource-constrained settings should be screened with a clinical algorithm in order to quickly identify those at high risk for TB. Patients reporting symptoms such as current cough, fever, weight loss or night sweats should be further evaluated for TB and other diseases. Persons living with HIV who screen positive for TB should undergo a specific Xpert MTB/RIF test as a primary diagnostic test. Xpert MTB/RIF assay is the only World Health Organization (WHO)-recommended diagnostic test that simultaneously detects mycobacterial infection and rifampicin resistance and is suitable for use at lower levels of the health system [47]. People who are unlikely to have active TB should be offered treatment for presumed latent TB infection [47].

ART initiation for those with a concomitant HIV-TB diagnosis is recommended to be offered as soon as possible and within 8-12 weeks of the TB diagnosis. The evidence reviewed for guidelines [48] suggests that individuals with a $\mathrm{CD}^{+}$cell count $<50$ cells per $\mathrm{mm}^{3}$ should be offered ART as soon as possible, within 2 weeks after TB treatment was started. This recommendation can be extended to those with a $\mathrm{CD}^{+}$cell count $<200$ cells per $\mathrm{mm}^{3}$ as this has been proven to reduce mortality. Evidence does not support early initiation of ART in individuals with central nervous system TB [49, 50].

Integrated TB/HIV care remains challenging. Further investments must be made to minimise the danger of nosocomial transmission of TB in HIV-infected patients. TB/HIV centres should have a patient-centred orientation with focus on treatment adherence in order to avoid treatment failure and relapse. All efforts must be done to maintain confidentiality for all data. Electronic tools, like electronic directly observed treatment, can be an alternative to face-to-face contacts in instances where geographical distance is an important factor. and should be used [48].

The next speaker, Carole Mitnick, presented the clinical trial landscape for MDR/XDR-TB, reviewing the current evidence and future perspectives. The cardiac safety of concomitant use of bedaquiline and delamanid was demonstrated in several trials focussing on the effect of these drugs on the QT interval. The results of the trials showed no additive effect of the two drugs on the corrected QT interval by Fredericia [51]. Another phase III clinical trial (Nix-TB) that enrolled patients with XDR-TB who had failed or were intolerant to MDR-TB regimens, evaluated the safety and efficacy of pretomanid, bedaquiline and linezolid [52]. The rate of favourable outcomes was 40 (88.9\%) out of 15, with a $95 \%$ confidence interval of 75.9-96.3\%. Most of the treatment-emergent adverse events were due to linezolid, and included peripheral neuropathy, anaemia, nausea, vomiting and headache [53].

A study by O'DonNell et al. [54] reviewed the effects of switching from antiretroviral efavirenz-containing regimens to twice-daily nevirapine in association with bedaquiline in patients with drug-resistant $\mathrm{TB}$ and HIV co-infection, highlighting a signal of low adherence to the new ART regimen leading to HIV viral failure, which could compromise the TB-specific benefits of bedaquiline in these patients. The need to establish whether kanamycin can safely and effectively be replaced by amikacin in new shortened regimens (without bedaquiline or delamanid) was emphasised in other studies reviewed in this session. Additional trials are also needed to compare shortened oral regimens with bedaquiline regimen.

Dr Mitnick's conclusions underlined the substantial progress with the three new drugs approved in the last 7 years but stressed the need to improve trial quality and to include vulnerable subgroups (e.g. pregnant women, substance users and homeless people). Reporting serious adverse events is critical for all drugs, not just for the "new" ones.

Cathy Hewison reviewed several observational studies on the efficacy and safety of new drugs for MDR/ XDR-TB. The endTB cohort is an observational trial focussing on prospective data collection and having the advantage of including patients not enrolled in randomised clinical trials (RCTs). This was the largest multicentric prospective study, which included 2727 patients from 17 countries (heterogenous population) with the purpose of determining the safety and efficacy of bedaquiline- or delamanid-containing regimens for MDR-TB, according to WHO recommendations. Patients received a regimen containing bedaquiline and/or delamanid between 1 April 2015 and 31 March 2018. Overall, there is no evidence of any major safety issue with either delamanid or bedaquiline. Of the 1109 patients included in the final analysis, 943 
(85\%) achieved sputum culture conversion within 6 months. Sputum culture conversion was defined as two consecutive negative cultures for $\mathrm{TB}$, collected $\geqslant 30$ days apart. Comparable sputum culture conversion rates were reported in other studies with bedaquiline (78\%) [55], delamanid (88\%) [56] or both (74\%) [57].

\section{Managing drug-resistant tuberculosis: from new guidelines to clinical practice}

Giovanni Battista Migliori presented the new recommendations of the American Thoracic Society, the Centers for Disease Control and Prevention, the ERS and the Infectious Diseases Society of America guidelines for MDR-TB. These guidelines were developed in the last 3 years by a team of experts including specialists in pulmonary medicine, infectious disease, pharmacokinetics, paediatrics, primary care and public health after multiple face-to-face meetings and weekly telephone calls. Strength of recommendations was formulated after reviewing all evidence, using the GRADE (Grading of Recommendations, Assessment, Development and Evaluation) approach. For each recommendation, the guideline panel considered and agreed on the quality of the evidence, balance of desirable and undesirable effects of compared alternative management options, and possible extent of resources. "Strong" and "weak" recommendations were defined for patients, clinicians and policy makers.

According to the guidelines, an effective MDR-TB treatment regimen should combine at least five drugs in the intensive phase and four drugs in the continuation phase of treatment. The recommended duration of intensive phase of treatment is 5-7 months after culture conversion. The recommended total treatment duration is 15-21 months after culture conversion for MDR-TB, and 15-24 months for XDR-TB and pre-XDR-TB.

Available oral drugs for MDR-TB treatment, in the order of strength of recommendations, are a later generation of fluoroquinolone (levofloxacin or moxifloxacin), bedaquiline, linezolid, clofazimine, cycloserine, ethambutol (when other more effective drugs cannot be added to achieve a total of five drugs in the regimen) and pyrazinamide (in regimens for patients with isoniazid-resistant TB). However, delamanid has a conditional recommendation according to the 2019 WHO Consolidated Guidelines of Drug-Resistant Tuberculosis Treatment for patients aged $\geqslant 3$ years on longer regimens. Injectables were also reviewed in the guidelines.

Other discussions referred to the necessity of consultation with a TB expert, a clinical service also active within the Global TB Network. Focus on rapid diagnosis and precision treatment includes newer genomic approaches such as the use of an automated Xpert drug resistant cartridge and the use of point-of-care portable battery-operated genomic tools (e.g. Xpert Edge and Xpert Omni) [58].

Therapeutic dose monitoring in the treatment of MDR-TB is used to identify patients with drug absorption problems, thereby needing dose adjustments in order to prevent poor response to TB treatment despite adherence.

Hospital admission requirements in Europe include clinical expertise on $\mathrm{TB}$ and MDR/XDR-TB management, quality-controlled laboratories, respiratory isolation capacity, infection control capability, and a patient-centred approach that includes psychological support and social services after discharge when needed $[59,60]$.

The guidelines support good management practices, recommending a TB consilium approach, prompt use of rapid diagnostic methods and drug sensitivity test-based regimens, management of adverse events, and adequate infection control [61-63].

\section{Therapeutic breakthrough Year in review}

The aim of this session was to highlight the key therapeutic developments that are likely to influence care and improve outcomes for adults and children with respiratory diseases. These therapies also have impact on long-term evolution of the underlying diseases, and on the number of future exacerbations and future respiratory infections.

The use of triple therapy versus dual bronchodilation and long-acting $\beta$-agonists (LABA)/inhaled corticosteroids (ICS) in COPD [64, 65] was reviewed by Mario Cazzola in the light of newly accumulating evidence from a couple of recently published network meta-analyses. Prof. Cazzola emphasised the importance of effectiveness data collected in real-life studies for the positioning of new therapies [66] and discussed the use of meta-analysis as a means of synthesising evidence across RCTs [67]. Compared to dual therapies, such as a LABA/long-acting muscarinic antagonists (LAMA) or LABA/ICS, triple therapy (ICS/LABA/LAMA) reduced the rates of moderate to severe exacerbations, and improved lung function and quality of life [68-72]; however, due to the increased risk of pneumonia for ICS-containing regimens [68] and to the fact that blood eosinophil count was found to be a significant effect modifier of triple 
therapy in the prevention of exacerbations $[69,71]$, the patients that are likely to benefit most from triple therapy are those with blood eosinophils counts $\geqslant 300$ cells per $\mu \mathrm{L}[69,73]$.

Keith Grimwood reviewed the therapeutic breakthrough of the year in the field of cystic fibrosis (CF) by presenting the results of the OPTIMIZE clinical trial [74]. The aim of this study was to assess whether the addition of azithromycin to tobramycin inhalation solution in children with CF and new-onset $P$. aeruginosa infection will reduce the risk of exacerbation, thus delaying $P$. aeruginosa recurrence. This was a multicentre, randomised, double-blind, placebo-controlled trial of 18 months duration, which included clinically stable children with $\mathrm{CF}$, aged 6 months to 18 years, with new positive cultures of $P$. aeruginosa within the previous 40 days. The risk of pulmonary exacerbation was reduced by $44 \%$ (hazard ratio 0.56 , 95\% CI $0.37-0.83 ; \mathrm{p}=0.004)$ and weight increased by $1.27 \mathrm{~kg}(95 \%$ CI $0.01-2.52 \mathrm{~kg} ; \mathrm{p}=0.046)$ in the azithromycin group as compared with the placebo group. No significant differences were seen in microbiological or other clinical or safety endpoints. These data support the use of azithromycin as a therapeutic option for children with CF and early $P$. aeruginosa infection, with no major safety concerns over the long-term use of azithromycin.

The therapeutic breakthrough of the year in pulmonary rehabilitation was presented by Elena Gimeno-Santos. A number of interventional studies on physical activity in patients with COPD published in the last 4 years [75-78] were reviewed. Physical activity is a complex and multifactorial behaviour [79], the evidence on its nonphysiological determinants being scarce. Urban Training is a multicomponent behavioural intervention that consists of physical training using walkable trails, located and adapted to the social environment of the patient, and other optional components for feedback, motivation, information and support. Dr Gimeno-Santos presented the results of a study aiming to assess the long-term efficacy and effectiveness of the Urban Training intervention to increase physical activity in COPD patients [80]. This was a RCT allocating COPD patients from primary and hospital settings 1:1 to usual care or Urban Training. Efficacy analysis (patients classified as adherent to the assigned intervention) showed an improvement in physical activity (steps per day) in the Urban Training group. Effectiveness analysis (patients completing the study at 12 months, including unwilling and self-reported nonadherent patients) showed no differences between groups. Leg muscle pain during walks was more frequently reported in Urban Training than usual care, without differences in any of the other adverse events. Dr Gimeno-Santos concluded her talk by briefly presenting other recently published studies on the duration, implementation and determinants of physical activity in COPD [81-84].

Paul Corris gave an overview on the role of extracorporeal assistance as a bridge and as support during lung transplantation. In the context of a constant increase in the number of organ donors and lung transplants, and improved pre-transplant treatment and noninvasive support for advanced disease, extracorporeal life support (ECLS) has become extremely relevant for patients referred for lung transplant procedure. Prof. Corris highlighted that ECLS has an established indication as a bridge to transplant in patients who have been evaluated and accepted for this procedure, and has potential indications in highly selected cases: 1) bridge to transplant decision in potentially eligible patients who have not yet been evaluated, and 2) bridge to recovery in patients with untreated or undertreated pulmonary hypertension or with a treatable cause of right ventricle failure $[85,86]$. ECLS has also become an established strategy for the support of patients with grade 3 primary graft dysfunction after lung transplantation. ECLS is contraindicated in patients with end-stage disease without a realistic chance for recovery [85].

\section{Concluding remarks}

The authors of this article wanted to highlight the importance of new therapeutic interventions in respiratory infections and TB. We also hope that this summarised information will be a useful tool for the readers to inform their daily practice clinical decisions.

Conflict of interest: None declared.

Author contributions: C. Chichirelo-Konstantynovych reported on the session "Fighting antimicrobial resistance: from prevention to antimicrobial stewardship", C. Calarasu reported on the sessions "Recent developments and future perspectives in the diagnosis and treatment of tuberculosis" and "Managing drug-resistant tuberculosis: from new guidelines to clinical practice", and S. Frent reported on the session "Therapeutic breakthrough Year in review". All authors reviewed and agreed on the content of the article.

\section{References}

1 European Centre for Disease Prevention and Control. Surveillance of antimicrobial resistance in Europe - Annual report of the European Antimicrobial Resistance Surveillance Network (EARS-Net) 2017. Stockholm, ECDC, 2018.

2 Waterer GW, Rello J, Wunderink RG. Management of community-acquired pneumonia in adults. Am J Respir Crit Care Med 2011; 183: 157-164. 
3 Aliberti S, Di Pasquale M, Zanaboni AM, et al. Stratifying risk factors for multidrug-resistant pathogens in hospitalized patients coming from the community with pneumonia. Clin Infect Dis 2012; 54: 470-478.

4 Aliberti S, Cilloniz C, Chalmers JD, et al. Multidrug-resistant pathogens in hospitalised patients coming from the community with pneumonia: a European perspective. Thorax 2013; 68: 997-999.

5 Di Pasquale MF, Sotgi G, Gramegna A, et al. Prevalence and etiology of community-acquired pneumonia in immunocompromised patients. Clin Infect Dis 2019; 68: 1482-1493.

6 Webb BJ, Sorensen J, Jephson A, et al. Broad-spectrum antibiotic use and poor outcomes in community-onset pneumonia: a cohort study. Eur Respir J 2019; 54: 1900057.

$7 \quad$ Fishman N. Antimicrobial stewardship. Am J Infect Control 2006; 34: Suppl. 1, S55-S63.

8 Deresinski S. Principles of antibiotic therapy in severe infections: optimizing the therapeutic approach by use of laboratory and clinical data. Clin Infect Dis 2007; 45: Suppl. 3, S177-S183.

9 Opal SM, Calandra T. Antibiotic usage and resistance: gaining or losing ground on infections in critically ill patients? JAMA 2009; 302: 2367-2368.

10 Kumar A, Roberts D, Wood KE, et al. Duration of hypotension before initiation of effective antimicrobial therapy is the critical determinant of survival in human septic shock. Crit Care Med 2006; 34: 1589-1596.

11 Rello J, Gallego M, Mariscal D, et al. The value of routine microbial investigation in ventilator-associated pneumonia. Am J Respir Crit Care Med 1997; 156: 196-200.

12 Alvarez-Lerma F. Modification of empiric antibiotic treatment in patients with pneumonia acquired in the intensive care unit. ICU-Acquired Pneumonia Study Group. Intensive Care Med 1996; 22: 387-394.

13 Ibrahim EH, Sherman G, Ward S, et al. The influence of inadequate antimicrobial treatment of bloodstream infections on patient outcomes in the ICU setting. Chest 2000; 118: 146-155.

14 Luna CM, Vujacich P, Niederman MS, et al. Impact of BAL data on the therapy and outcome of ventilator-associated pneumonia. Chest 1997; 111: 676-685

15 Garnacho-Montero J, Garcia-Garmendia JL, Barrero-Almodovar A, et al. Impact of adequate empirical antibiotic therapy on the outcome of patients admitted to the intensive care unit with sepsis. Crit Care Med 2003; 31: 2742-2751.

16 Vallés J, Rello J, Ochagavía A, et al. Community-acquired bloodstream infection in critically ill adult patients: impact of shock and inappropriate antibiotic therapy on survival. Chest 2003; 123: 1615-1624.

17 O'Neill J. Tackling Drug-resistant Infections Globally: Final Report and Recommendations. London, Wellcome Trust and HM Government, 2016.

18 Titmarsh S. Tackling the growing problem of antifungal resistance. Prescriber 2018; 29: 24-27.

19 Fleming-Dutra KE, Hersh AL, Shapiro DJ, et al. Prevalence of inappropriate antibiotic prescriptions among US ambulatory care visits, 2010-2011. JAMA 2016; 315: 1864-1873.

20 Livermore DM. $\beta$-Lactamases in laboratory and clinical resistance. Clin Microbiol Rev 1995; 8: 557-584.

21 Klein EY, Van Boeckel TP, Martinez EM, et al. Global increase and geographic convergence in antibiotic consumption between 2000 and 2015. Proc Natl Acad Sci USA 2018; 115: E3463-E3470.

22 Collignon P, Beggs JJ, Walsh TR, et al. Anthropological and socioeconomic factors contributing to global antimicrobial resistance: a univariate and multivariable analysis. Lancet Planet Health 2018; 2: e398-e405.

23 Jain S, Self WH, Wunderink RG, et al. Community-acquired pneumonia requiring hospitalization among U.S adults. N Engl J Med 2015; 373: 415-427.

24 Ieven $\mathrm{M}$, Coenen $\mathrm{S}$, Loens $\mathrm{K}$, et al. Aetiology of lower respiratory tract infection in adults in primary care: a prospective study in 11 European countries. Clin Microbiol Infect 2018; 24: 1158-1163.

25 Havers FP, Hicks LA, Chung JR, et al. Outpatient antibiotic prescribing for acute respiratory infections during influenza seasons. JAMA Netw Open 2018; 1: e180243.

26 Brendish NJ, Malachira AK, Armstrong L, et al. Routine molecular point-of-care testing for respiratory viruses in adults presenting to hospital with acute respiratory illness (ResPOC): a pragmatic, open-label, randomised controlled trial. Lancet Respir Med 2017; 5: 401-411.

27 Cooke J, Butler C, Hopstaken R, et al. Narrative review of primary care point-of-care testing (POCT) and antibacterial use in respiratory tract infection (RTI). BMJ Open Respir Res 2015; 2: e000086.

28 Assicot $\mathrm{M}$, Gendrel D, Carsin $\mathrm{H}$, et al. High serum procalcitonin concentrations in patients with sepsis and infection. Lancet 1993; 341: 515-518.

29 Schuetz P, Wirz Y, Sager R, et al. Procalcitonin to initiate or discontinue antibiotics in acute respiratory tract infections. Cochrane Database Syst Rev 2017; 10: CD007498.

30 Rhee C. Using Procalcitonin to Guide Antibiotic Therapy. Open Forum Infect Dis 2016; 4: ofw249.

31 Wittermans E, Vestjens SMT, Bos WJW, et al. The extent of microbiological testing is associated with alteration of antibiotic therapy in adults with community-acquired pneumonia. Eur J Clin Microbiol Infect Dis 2019; 38 $1359-1366$.

32 Gal M, Francis NA, Hood K, et al. Matching diagnostics development to clinical need: Target product profile development for a point of care test for community-acquired lower respiratory tract infection. PLoS One 2018; 13 : e0200531.

33 Sikaris KA. Enhancing the clinical value of medical laboratory testing. Clin Biochem Rev 2017; 38: 107-114.

34 Eurostat. Healthcare expenditure statistics. https://ec.europa.eu/eurostat/statistics-explained/index.php/Healthcare expenditure_statistics. Date last updated: July 2019.

35 Laxminarayan R, Duse A, Wattal C, et al. Antibiotic resistance-the need for global solutions. Lancet Infect Dis 2013; 13: 1057-1098.

36 Little P, Stuart B, Moore M, et al. Amoxicillin for acute lower-respiratory-tract infection in primary care when pneumonia is not suspected: a 12-country, randomised, placebo-controlled trial. Lancet Infect Dis 2013; 13: 123-129.

37 Little P, Stuart B, Francis N, et al. Effects of internet-based training on antibiotic prescribing rates for acute respiratory-tract infections: a multinational, cluster, randomised, factorial, controlled trial. Lancet 2013; 382 $1175-1182$.

38 Keitel K, Kagoro F, Samaka J, et al. A novel electronic algorithm using host biomarker point-of-care tests for the management of febrile illnesses in Tanzanian children (e-POCT): a randomized, controlled non-inferiority trial. PLoS Med 2017; 14: e1002411.

39 Johnson AP, Ashiru-Oredope D, Beech E. Antibiotic stewardship initiatives as part of the UK 5-year antimicrobial resistance strategy. Antibiotics (Basel) 2015; 4: 467-479. 
40 Karanika S, Paudel S, Grigoras C, et al. Systematic review and meta-analysis of clinical and economic outcomes from the implementation of hospital-based antimicrobial stewardship programs. Antimicrob Agents Chemother 2016; 60: 4840-4852.

41 Baur D, Gladstone BP, Burkert F, et al. Effect of antibiotic stewardship on the incidence of infection and colonisation with antibiotic-resistant bacteria and Clostridium difficile infection: a systematic review and meta-analysis. Lancet Infect Dis 2017; 17: 990-1001.

42 Schuts EC, Hulscher MEJL, Mouton JW, et al. Current evidence on hospital antimicrobial stewardship objectives: a systematic review and meta-analysis. Lancet Infect Dis 2016; 16: 847-856.

43 Timbrook TT, Morton JB, McConeghy KW, et al. The effect of molecular rapid diagnostic testing on clinical outcomes in bloodstream infections: a systematic review and meta-analysis. Clin Infect Dis 2017; 64: 15-23.

44 Pulcini C, Binda F, Lamkang AS, et al. Developing core elements and checklist items for global hospital antimicrobial stewardship programmes: a consensus approach. Clin Microbiol Infect 2019; 25: 20-25.

45 Naidoo K, Gengiah S, Yende-Zuma N, et al. Addressing challenges in scaling up TB and HIV treatment integration in rural primary healthcare clinics in South Africa (SUTHI): a cluster randomized controlled trial protocol. Implement Sci 2017; 12: 129.

46 McCarthy K, Goemaere E, Wilkinson L, et al. A practical guide for TB and HIV service integration at primary health care facilities. www.inpracticeafrica.com/ /media/Guidelines/Practical_Guide_TB HIV.pdf. Date last accessed: 11 November 2019.

47 World Health Organization. Systematic screening for active tuberculosis: principles and recommendations. www. who.int/tb/publications/tbscreening/en. Date last accessed: 11 November 2019.

48 British HIV Association. British HIV Association guidelines for the management of tuberculosis in adults living with HIV 2018 (2019 interim update). www.bhiva.org/file/5c485f3dc7c17/BHIVA-TB-guidelines.pdf. Date last accessed: 5 November 2019.

49 Blanc FX, Sok T, Laureillard D, et al. Earlier versus later start of antiretroviral therapy in HIV-infected adults with tuberculosis. N Engl J Med 2011; 365: 1471-1481.

50 Pathmanathan I, Pasipamire M, Pals S, et al. High uptake of antiretroviral therapy among HIV-positive TB patients receiving co-located services in Swaziland. PLoS One 2018; 13: e0196831.

51 The Deliberate Trial (ACTG A5343) Oral abstract 84. Presented at CROI 2019. http://i-base.info/htb/35916. Date last accessed: 5 November 2019.

52 Pretomanid and BPaL Regimen for Treatment of Highly-Resistant Tuberculosis, TB Alliance, Antimicrobial Drugs Advisory Committee, June 6, 2019. www.fda.gov/media/128001/download. Date last accessed: 27 December 2019.

53 Food and Drug Administration. FDA Briefing Document, Pretomanid tablet, $200 \mathrm{mg}$, Meeting of the AMDAC, June 06, 2019. www.fda.gov/media/127592/download. Date last accessed: 1 November 2019.

54 O'Donnell MR, Padayatchi N, Daftary A, et al. Antiretroviral switching and bedaquiline treatment of drug-resistant tuberculosis HIV co-infection. Lancet HIV 2019; 6: e201-e204.

55 Mbuagbaw L, Guglielmetti L, Hewison C, et al. Outcomes of bedaquiline treatment in patients with multidrug-resistant tuberculosis. Emerging Infect Dis 2019; 25: 936-943.

56 von Groote-Bidlingmaier F, Patientia R, Sanchez E, et al. Efficacy and safety of delamanid in combination with an optimised background regimen for treatment of multidrug-resistant tuberculosis: a multicentre, randomised double-blind, placebo-controlled, parallel group phase 3 trial. Lancet Respir Med 2019; 7: 249-259.

57 Ferlazzo G, Mohr E, Laxmeshwar C, et al. Early safety and efficacy of the combination of bedaquiline and delamanid for the treatment of patients with drug-resistant tuberculosis in Armenia, India, and South Africa: a retrospective cohort study. Lancet Infect Dis 2018; 18: 536-544.

58 Sohn $\mathrm{H}$, Kasaie $\mathrm{P}$, Kendall $\mathrm{E}$, et al. Informing decision-making for universal access to quality tuberculosis diagnosis in India: an economic-epidemiological model. BMC Med 2019; 17: 155.

59 Migliori GB, Hopewell PC, Blasi F, et al. Improving the TB case management: the International Standards for Tuberculosis care. Eur Respir J 2006; 28: 687-690.

60 Borisov S, Danila E, Maryandyshev A, et al. Surveillance of adverse events in the treatment of drug-resistant tuberculosis: first global report. Eur Respir J 2019; 54: 1901522.

61 Migliori GB, D’Ambrosio L, Centis R, et al. Guiding Principles to Reduce Tuberculosis Transmission in the WHO European Region. www.euro.who.int/_data/assets/pdf_file/0008/377954/ic-principles-eng.pdf

62 World Health Organization. WHO guidelines on tuberculosis infection prevention and control, 2019 update. www.who.int/tb/publications/2019/guidelines-tuberculosis-infection-prevention-2019/en/. Date last accessed: 11 November 2019.

63 Migliori GB, Nardell E, Yedilbayev A, et al. Reducing tuberculosis transmission: a consensus document from the World Health Organization Regional Office for Europe. Eur Respir J 2019; 53: 1900391.

64 Singh D, Agusti A, Anzueto A, et al. Global Strategy for the Diagnosis, Management, and Prevention of Chronic Obstructive Lung Disease: the GOLD science committee report 2019. Eur Respir J 2019; 53: 1900164.

65 Woodcock A, Boucot I, Leather DA, et al. Effectiveness versus efficacy trials in COPD: how study design influences outcomes and applicability. Eur Respir J 2018; 51: 1701531.

66 Gurevitch J, Koricheva J, Nakagawa S, et al. Meta-analysis and the science of research synthesis. Nature 2018; 555: $175-182$

67 Zheng Y, Zhu J, Liu Y, et al. Triple therapy in the management of chronic obstructive pulmonary disease: systematic review and meta-analysis. BMJ 2018; 363: k4388.

68 Cazzola M, Rogliani P, Calzetta L, et al. Triple therapy versus single and dual long-acting bronchodilator therapy in COPD: a systematic review and meta-analysis. Eur Respir J 2018; 52: 1801586.

69 Calzetta L, Cazzola M, Matera MG, et al. Adding a LAMA to ICS/LABA therapy: a meta-analysis of triple combination therapy in COPD. Chest 2019; 155: 758-770.

70 Zayed Y, Barbarawi M, Kheiri B, et al. Triple versus dual inhaler therapy in moderate-to-severe COPD: a systematic review and meta-analysis of randomized controlled trials. Clin Respir J 2019; 13: 413-428.

71 Suissa S, Ariel A. Triple therapy trials in COPD: a precision medicine opportunity. Eur Respir J 2018; 52 : 1801848.

72 Pascoe S, Barnes N, Brusselle G, et al. Blood eosinophils and treatment response with triple and dual combination therapy in chronic obstructive pulmonary disease: analysis of the IMPACT trial. Lancet Respir Med 2019; 7: 745-756 
73 Mayer-Hamblett N, Retsch-Bogart G, Kloster M, et al. Azithromycin for early Pseudomonas infection in cystic fibrosis. The OPTIMIZE randomized trial. Am J Respir Crit Care Med 2018; 198: 1177-1187.

74 Mendoza L, Horta P, Espinoza J, et al. Pedometers to enhance physical activity in COPD: a randomised controlled trial. Eur Respir J 2015; 45: 347-354.

75 Moy M, Martinez C, Kadri R, et al. Long-term effects of an internet-mediated pedometer-based walking program for chronic obstructive pulmonary disease: randomized controlled trial. J Med Internet Res 2016; 18: e215.

76 Altenburg WA, ten Hacken NH, Bossenbroek L, et al. Short- and long-term effects of a physical activity counselling programme in COPD: a randomized controlled trial. Respir Med 2015; 109: 112-121.

77 Demeyer H, Louvaris Z, Frei A, et al. Physical activity is increased by a 12-week semiautomated telecoaching programme in patients with COPD: a multicentre randomised controlled trial. Thorax 2017; 72: 415-423.

78 Bauman AE, Reis RS, Sallis JF, et al. Correlates of physical activity: why are some people physically active and others not? Lancet 2012; 380: 258-271.

79 Arbillaga-Etxarri A, Gimeno-Santos E, Barberan-Garcia A, et al. Long-term efficacy and effectiveness of a behavioural and community-based exercise intervention (Urban Training) to increase physical activity in patients with COPD: a randomised controlled trial. Eur Respir J 2018; 52: 1800063.

80 Wootton SL, Hill K, Alison JA, et al. Effects of ongoing feedback during a 12-month maintenance walking program on daily physical activity in people with COPD. Lung 2019; 197: 315-319.

81 Loeckx M, Rabinovich RA, Demeyer $\mathrm{H}$, et al. Smartphone-based physical activity telecoaching in chronic obstructive pulmonary disease: mixed-methods study on patient experiences and lessons for implementation. JMIR Mhealth Uhealth 2018; 6: e200.

82 Koreny M, Demeyer H, Arbillaga-Etxarri A, et al. Determinants of study completion and response to a 12-month behavioral physical activity intervention in chronic obstructive pulmonary disease: A cohort study. PLoS ONE; 14: e0217157.

83 Koolen EH, van Hees HW, van Lummel RC, et al. "Can do" versus "do do": a novel concept to better understand physical functioning in patients with chronic obstructive pulmonary disease. J Clin Med 2019; 8: E340.

84 Hoeper MM, Benza RL, Corris P, et al. Intensive care, right ventricular support and lung transplantation in patients with pulmonary hypertension. Eur Respir J 2019; 53: 1801906.

85 Moreno Garijo J, Cypel M, McRae K, et al. The evolving role of extracorporeal membrane oxygenation in lung transplantation: implications for anesthetic management. J Cardiothorac Vasc Anesth 2019; 33: 1995-2006.

86 Geube M, Anandamurthy B, Yared JP. Perioperative management of the lung graft following lung transplantation. Crit Care Clin 2019; 35: 27-43. 